\title{
High-fluence and high-flux performance characteristics of the superconducting Magnum-PSI linear plasma facility
}

H.J.N. van Eck*, G.R.A. Akkermans, S. Alonso van der Westen, D.U.B. Aussems, M. van Berkel, S. Brons, I.G.J. Classen, H.J. van der Meiden, T.W. Morgan, M.J. van de Pol, J. Scholten, J.W.M. Vernimmen, E.G.P. Vos and M.R. de Baar

DIFFER - Dutch Institute for Fundamental Energy Research, De Zaale 20, 5612 AJ Eindhoven, the Netherlands

*Corresponding author : $\underline{\text { h.j.n.vaneck@differ.nl }}$

\section{Highlights}

- The Magnum-PSI facility is available for plasma-material interaction studies.

- Magnum-PSI is capable to reach relevant plasma parameters for the ITER divertor.

- Particle fluxes over $10^{25} \mathrm{~m}^{-2} \mathrm{~s}^{-1}$ and heat fluxes of up to $50 \mathrm{MWm} \mathrm{m}^{-2}$ are obtained.

- Particle fluences of up to $10^{30}$ particles $\mathrm{m}^{-2}$ have been achieved.

- Linear regression and artificial neural network analysis have been applied.

\section{Abstract}

The Magnum-PSI facility is unique in its ability to produce and even exceed the heat and particle fluxes expected in the divertor of a fusion reactor, combined with good access to the plasma-material interaction region for diagnostics and relatively easy sample manipulation. In addition, it is possible to study the effects of transient heat loads on a plasma-facing surface, similar to those expected during so called Edge Localized Modes. By virtue of a newly installed superconducting magnet, Magnum-PSI can now maintain these conditions for hours on end for truly long term tests of candidate plasma facing materials. The electron density and temperature in the plasma beam center as a function of different magnetic fields up to $1.6 \mathrm{~T}$, gas flow and source current are determined: particle fluxes greater than $10^{25} \mathrm{~m}^{-2} \mathrm{~s}^{-1}$ and heat fluxes of up to $50 \mathrm{MWm}^{-2}$ are obtained. Linear regression and artificial neural network analysis have been used to gain insight in the general behavior of plasma conditions as a function of these machine settings. The plasma conditions during transient plasma heat loading have also been determined. These capabilities are now being exploited to reach fluence of up to $10^{30}$ particles $\mathrm{m}^{-2}$ at ITER-relevant conditions, equivalent to a significant fraction of the divertor service lifetime for the first time.

\section{Keywords}

Linear plasma devices; Plasma-surface interactions; High heat flux; Edge Localized Modes; Divertor; Artificial neural network analysis 


\section{Introduction}

The development of a solution for the removal of heat and particles from the reactor is a key area of presentday fusion research, as it determines the performance, lifetime and safety of future fusion power plants. An important contribution to this research is provided by linear plasma generators, which can address the complex physics of plasma wall interaction in a systematic way, with controlled plasma parameters, flexible target geometry and good diagnostic access. The linear plasma facility Magnum-PSI is capable of exposing materials to steady state plasma conditions similar to those foreseen in the ITER and DEMO divertor strike zones: $10^{23}$ $10^{25} \mathrm{~m}^{-2} \mathrm{~s}^{-1}$ hydrogen plasma flux densities at an electron and ion temperature of 1-5 eV. It utilizes a superconducting magnet to generate an axial magnetic field up to $2.5 \mathrm{~T}$ which confines the high density, low temperature plasma produced by a wall stabilized dc cascaded arc [1] into an intense magnetized plasma beam (FWHM $\approx 10-25 \mathrm{~mm}$ ) directed onto a target. In this way, extremely high ion fluxes $\left(>10^{25} \mathrm{~m}^{-2} \mathrm{~s}^{-1}\right)$ with heat fluxes up to $50 \mathrm{MWm}^{-2}$ can be achieved in steady state operation. This enables lifetime studies of fusion materials in a lab environment for the first time within a reasonable time frame (i.e. hours). Extremely high fluences over $10^{30}$ $\mathrm{D}^{+} \mathrm{m}^{-2}$ have already been achieved with steady state power densities over $10 \mathrm{MWm}^{-2}$. In addition, the machine is capable of reproducing the transient heat and particle loads, with powers up to 1 $\mathrm{GWm}^{-2}$, as they occur during Edge Localized Modes (ELM) [2]. This combined plasma and heat loading can be used to study the synergistic damaging effects on a target material. Transient plasma loading can also be compared to laser loading using a high-power welding laser (LASAG FLS 352-302) to generate 0.1-3 ms heating pulses on the target. As such, Magnum-PSI, together with other linear plasma generators and laboratory heat facilities [3] are deemed essential to predict plasma facing component (PFC) performance at high particle fluence $\left(>10^{27} \mathrm{D}^{+} \mathrm{m}^{-2}\right)$ and high number of thermal cycles ( $>10^{6}$ ELM-like events) within the EUROfusion consortium [4].

These operations at high magnetic field strengths and over long time durations are achievable since the installation of the superconducting magnet at the start of 2017. Since then, an examination of the new capabilities of the machine was carried out. In this contribution, the operational regime and experimental capabilities of Magnum-PSI are presented.

\section{Experimental setup}

To reach the required ion flux, a certain gas flow is needed, depending on the ionization degree of the plasma source. This gas flow can be as high as 20 standard liter per minute $(\mathrm{s} / \mathrm{m})$. However, the neutral pressure near the target material should be almost entirely due to ions which are neutralized at the target plate and not due to neutrals coming directly from the source. This is achieved by using a three stage differentially pumped vacuum system [5] which minimizes the neutral influx to the target region while leaving the plasma beam undisturbed. This results in a neutral pressure in the target region during plasma exposure between 0.1 and $0.5 \mathrm{~Pa}$, depending 
on the machine settings. The base pressure without gas injection is $2 \times 10^{-4} \mathrm{~Pa}$. In Figure 1 a schematic overview of the Magnum-PSI vacuum vessel is given. The three water cooled vacuum chambers are enclosed by the superconducting magnet (not shown). The first chamber as seen from the right holds the movable plasma source. The movement of the plasma source makes it possible to change its position with respect to the first skimmer. The magnetized plasma beam flows through the first skimmer into the beam dump chamber where a water cooled beam dump can be placed between the plasma beam and the target. In this way the length of the target exposure can be set without switching off the plasma source or adjustment of the source parameters to the desired settings without exposing the target surface. In the third chamber the target material is exposed to the plasma beam. Each chamber is pumped with a separate pump combination consisting of two roots pumps and a backing pump with variable pump speeds up to $20.000 \mathrm{~m}^{3} \mathrm{~h}^{-1}$. Additionally, seeding gases $\left(H_{2}, D_{2}, \mathrm{He}, \mathrm{N}_{2}\right.$, $\mathrm{Ne}, \mathrm{Ar} . .$.$) can now be introduced to this third chamber to adjust the neutral background pressure and$ composition at will, enabling detachment and seeding experiments [6]. By using this differential pumping scheme, the neutral pressure in the target region is low enough that the dominant source of neutrals in the final chamber is dominated by recycling from the target [7] and the plasma beam is transported from the source to the target with minimal losses.

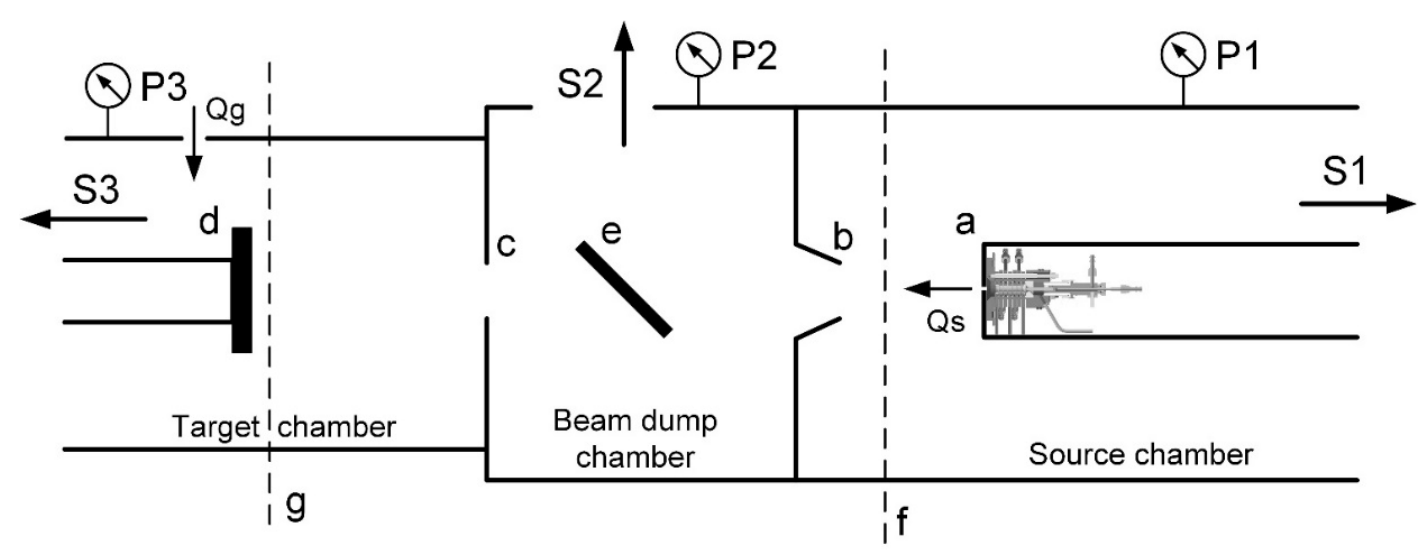

Figure 1. Schematic overview of the differentially pumped Magnum-PSI experiment inside the superconducting magnet. Shown are (from right to left), the source chamber, beam dump chamber and target chamber each pumped by their own pumping station (S1, S2 and S3). The source chamber holds the plasma source which is mounted inside a movable water cooled tube (a). The magnetized beam flows through the skimmers ( $b$ and $c$ ) from the source to the target (d) while most of the neutrals are scraped off and pumped away. A beam dump (e) can be placed between the plasma beam and the target. Thomson scattering yields electron density and temperature profiles near the source (f) and directly in front of the target (g). Gas inlet from the source and gas puffing are indicated with Qs and Qg respectively.

The magnetic field is generated by a superconducting magnet consisting of five $\mathrm{NbTi}$ superconducting solenoids wound on a $2.5 \mathrm{~m}$ long stainless steel coil former positioned in a cryostat offering a $1.25 \mathrm{~m}$ warm bore [8]. The coils generate a plateau shaped magnetic field adjustable up to $2.5 \mathrm{~T}$ while the distance between the coils allows 
for 16 room temperature view-ports. The coils are cooled with liquid helium using a re-condensing system operated with cryocoolers, while the magnet system can be adjusted between any chosen field at will using high-temperature superconducting current leads. The superconducting magnet makes extremely high fluence exposures possible while the large warm bore leaves enough room for diagnostics.

A key benefit for linear plasma devices is their good accessibility and large number of diagnostic windows which allows for many different plasma and surface diagnostics to be installed. The most important plasma diagnostic is Thomson scattering (TS), which determines the electron density and temperature profiles downstream from the source exit and directly in front of the target with a spatial resolution of $1.6 \mathrm{~mm}$ [9]. Recently several new diagnostics have been installed. The ion temperature and axial/rotational velocity of the plasma beam can be measured with Collective Thomson scattering (CTS) [10], while a 4-channel resistive foil bolometer is now available for quantitative measurement of the plasma radiated power [11]. A new quartz crystal mass balance (QCMB, Inficon front load single sensor/STM-2 PN 074-613-P1D) can measure deposition rates of material eroding from the target. Fast voltage and current measurements of the plasma source are now also available, enabling determination of changes during transient events such as the ELM-like simulations.

Information on the composition of the plasma in front of the target is obtained from single channel survey optical emission spectroscopy (Avantes AvaSpec-2048-USM2-RM) covering the range 299-950 nm, while spatially resolved spectroscopy can be performed with the aid of a $1 \mathrm{~m}$ Jarrell-Ash spectrometer with a flexible spectral range $175 \mathrm{~nm}$ wide. Cooling water calorimetry can be carried out to measure the power deposited on the target by the plasma beam, while a fast infrared camera (FLIR SC7500MB) together with a single-channel emissivity independent multi-wavelength pyrometer (FAR-Associate Spectro Pyrometer FMPI) can be used to determine the target surface temperature with a spatial resolution of around $300 \times 300 \mu \mathrm{m}$ pixel ${ }^{-1}$. Fast events up to $1 \mathrm{MHz}$ can be captured by a fast visible light camera (Phantom).

Magnum-PSI is capable of handling large targets, up to an area of $0.12 \times 0.6 \mathrm{~m}^{2}$ and weight of $20 \mathrm{~kg}$. The Large Target Holder is highly flexible and can be tilted to very low angles $\left(<3^{\circ}\right)$, replicating the incidence angles of plasma onto the target material in a fusion reactor. Additionally a second target holder is available which can accommodate up to five samples of dimensions $<40 \mathrm{~mm}$ diameter and $10 \mathrm{~mm}$ thickness, exposing each in turn at normal incidence to the plasma. This Multi-target Holder enables fast sample throughput without breaking vacuum to exchange samples each time. After plasma exposure, the target holder is retracted to the Target Exchange and Analysis Chamber (TEAC). Here, the connection to an ion beam line enables ion beam analysis to locally probe material composition and obtain elemental depth profiles without breaking vacuum, for instance to investigate fuel retention in nuclear fusion reactor walls. Laser-Induced Breakdown Spectroscopy (LIBS) is also available in the TEAC to determine elemental concentration as a function of depth post-mortem in vacuo [12]. 


\section{Operational space characterization}

\subsection{Experimental results}

The measurements presented here are performed with an electrically floating target, typically made of $\mathrm{W}$, in the perpendicular position with respect to the plasma beam using the Multi-target holder. Only hydrogen plasmas in steady state are considered. Electron density $n_{\mathrm{e}}$ and electron temperature $T_{\mathrm{e}}$ profiles have been measured with TS $20-50 \mathrm{~mm}$ in front of the target while the source is kept at its standard position (131 $\mathrm{mm}$ in front of the first skimmer). The accuracy of $n_{\mathrm{e}}$ and $T_{\mathrm{e}}$ is $3 \%$ and $6 \%$ respectively at $n_{\mathrm{e}}=1.5 \times 10^{19} \mathrm{~m}^{-3}$ for an accumulation time of $3 \mathrm{~s}$. Discharges on different days using the same machine settings were found to reproduce the same $n_{e}$ and $T_{e}$ within typically $8 \%$ and $6 \%$, respectively. The magnetic field $B(T)$, gas flow $\Gamma(\mathrm{s} / \mathrm{m})$ and source current I (A) were varied while the pump speeds were always kept in the range $80-100 \%$. Within this range, variations in pump speed have only a minor effect on the plasma conditions. So far, magnetic fields up to $1.6 \mathrm{~T}$ have been commissioned.

Figure 2 shows a scatter plot of $n_{\mathrm{e}}$ and $T_{\mathrm{e}}$ in the beam center, with color indicating the setting of (a) magnetic field, (b) gas flow, and (c) source current. Values up to $n_{\mathrm{e}}=10^{21} \mathrm{~m}^{-3}$ and $T_{\mathrm{e}}=4.7 \mathrm{eV}$ were separately achieved. Lines of constant ion flux (solid) and power flux (dashed) are given, which show that particle fluxes of up to $10^{25}$ $\mathrm{m}^{-2} \mathrm{~s}^{-1}$ and heat fluxes of up to $50 \mathrm{MWm}^{-2}$ are obtained. The ion flux to the target is calculated from the Bohm flux [13]:

$$
\Gamma_{i}=0.5 n_{e} c_{s}=0.5 \mathrm{n}_{\mathrm{e}}\left[\left(k_{\mathrm{B}} T_{e}+\gamma k_{B} T_{i}\right) / m_{i}\right]^{1 / 2},
$$

assuming adiabatic flow with isotropic pressure $(\gamma=5 / 3)$, equilibration between electrons and ions $\left(T_{\mathrm{e}}=T_{\mathrm{i}}\right)$, and a density drop of a factor 0.5 in the pre-sheath, i.e. between the measurement location and the target. The heat flux to the target is calculated by:

$$
q=\Gamma_{i}\left[\left(2.5 k_{B} T_{e}-e V_{s}-e V_{p s}\right)\left(1-R_{i, E}\right)+2 k_{B} T_{e}\left(1-R_{e, E}\right)+\chi_{i}+\chi_{r}\left(1-R_{n}\right)\right]=\Gamma_{i}\left[5.03 k_{B} T_{e}+e 14.5(\mathrm{eV})\right],
$$

where $V_{s}=k T_{e} / e \ln \left(4 c_{i} / c_{e}\right)=0.5 \ln \left[\left(2 \pi m_{e} / m_{i}\right)\left(1+\gamma T_{i} / T_{e}\right)\right.$ is the sheath potential drop for which we assume $c_{i}=c_{s}$, and the pre-sheath potential drop $V_{p s}=\ln (0.5) k T_{e} / e, \chi_{i}=13.6 \mathrm{eV}, \chi_{r}=2.2 \mathrm{eV}$ are the ionization and dissociation energy of hydrogen, and $R_{i, E}=0.40$ and $R_{e, E}=0.15$ are the ion and electron energy reflection coefficients and $R_{n}$ $=0.40$ the neutral reflection coefficient for $\mathrm{W}$ [14]. The arrows indicate the trends for increasing the depicted parameter while keeping the others constant, e.g. an increasing magnetic field leads to higher $n_{\mathrm{e}}$ and $T_{\mathrm{e}}$ values. More details are given in Sec. 3.2. 

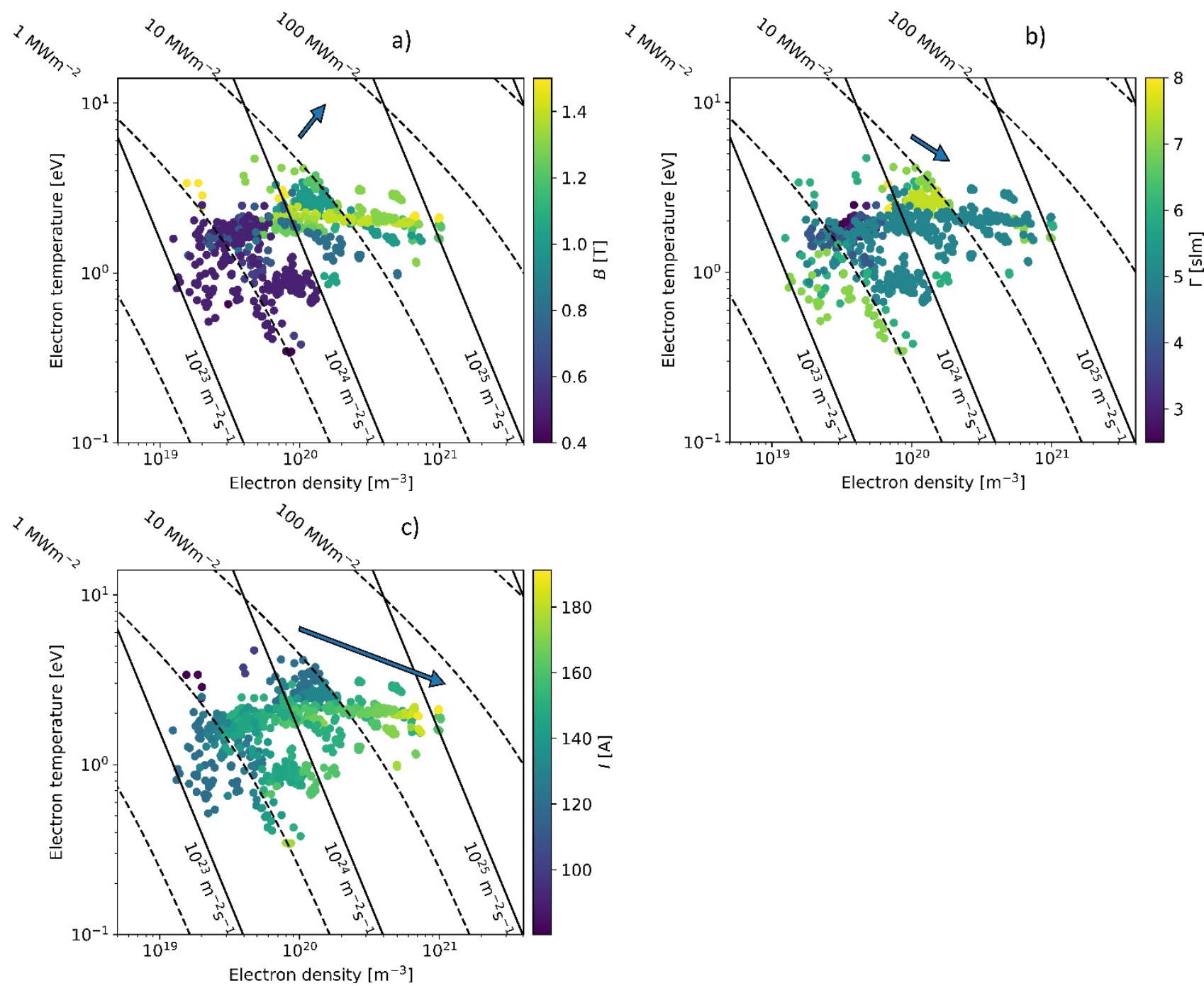

Figure 2. $n_{\mathrm{e}}$ and $T_{\mathrm{e}}$ in the beam center for various magnetic fields (a), gas flows (b) and source currents (c) shown in color. Lines of constant ion flux (solid) and power flux (dashed) are given. Values up to $n_{\mathrm{e}}=10^{21} \mathrm{~m}^{-3}, T_{\mathrm{e}}=4.7 \mathrm{eV}, \Gamma_{i}=10^{25} \mathrm{~m}^{-2} \mathrm{~s}^{-1}$, and $q=50 \mathrm{MWm}^{-2}$ were separately achieved. Arrows indicate the trend of change in temperature and density when the depicted machine setting is increased, showing that increased magnetic field raises both $n_{\mathrm{e}}$ and $T_{\mathrm{e}}$, while an increase of either gas flow or source current causes higher $n_{\mathrm{e}}$ but lower $T_{\mathrm{e}}$. The length of the arrow shows the relative strength of the dependence on each machine setting.

\subsection{Linear Regression Analysis}

In order to gain insight in the general behavior of plasma conditions as a function of the main machine settings, we fitted the data using a scaling law of the form:

$$
\begin{aligned}
& n_{\mathrm{e}}=\left.C_{n} \Gamma^{a_{\Gamma, n}} B^{a_{B, n}}\right|^{a^{a}, \mathrm{n},}, \\
& T_{\mathrm{e}}=\left.C_{T} \Gamma^{a_{\Gamma, T}} B^{a_{B, T}}\right|^{a_{1, T} .} .
\end{aligned}
$$

Such a scaling law can disentangle the effect of the different machine settings in a database where all three parameters change across varying data points. To this end, the plasma conditions and machine settings are first 
normalised by their respective units. Then, the logarithm of both sides of Eq. 3a,b is taken, which - when applied to all points in the database - yields two linear systems of equations. These are solved in linear least squares fashion to obtain the scaling exponents $a_{i, j}$ and constants $C_{j}$. The results are:

$$
\begin{aligned}
& n_{\mathrm{e}}=10^{7.9 \pm 0.4} \Gamma^{1.3 \pm 0.1} B^{0.91 \pm 0.05} I^{5.2 \pm 0.2,} \\
& T_{\mathrm{e}}=10^{4.3 \pm 0.2} \Gamma^{-0.71 \pm 0.05} B^{0.96 \pm 0.03} I^{-1.6 \pm 0.1} .
\end{aligned}
$$

The exponents in Eqs. 4 were used in Figure 2 to determine the length and direction of the arrows indicating the single-parameter trends. For $B$ and $I$, the arrows compare relatively clearly with the observable trends in the raw data, but for $\Gamma$, the trend found by the scaling law is completely hidden in the raw data by changes in $B$ and I.

The quality of the scaling law fit is assessed in Figure 3, where the measured $n_{\mathrm{e}}(\mathrm{a})$ and $T_{\mathrm{e}}$ (b) are plotted as a function of the values predicted by the scaling law. The fits are able to reproduce the experimental data within a factor $10^{ \pm 0.22}(+64 \% /-39 \%)$ for density and $10^{ \pm 0.12}(+30 \% /-23 \%)$ for temperature $(\mathrm{rms})$, with $\mathrm{R}^{2}$ values of 0.75 and 0.65 for $n_{\mathrm{e}}$ and $T_{\mathrm{e}}$ respectively. This larger error compared to the reproducibility and accuracy uncertainties of the TS measurements is most likely due to the fact that different regimes in the operating space may exhibit locally different proportionalities between machine settings and plasma parameters. For example, a specific atomic or molecular process may dominate the plasma in one region of parameter space but can be absent in other regions. Different processes will respond differently to changes in machine settings as is common with scaling laws. Hence, the scaling laws capture the overall performance of our machine but not individual regimes. Moreover, as scaling laws combine different physical dependencies, specific regime dependent physical dependencies can be lost. This is reflected by analyzing the difference between the ionization regime and recombination regime for the same data set.

If the peak electron temperature is below $1 \mathrm{eV}$ the recombination process generally dominates and if it is larger than $1 \mathrm{eV}$ we assume that ionization is the dominant process. Hence, using this criteria, we can split the data into two data sets, separating approximately ionization from recombination. This resulted in $80 \%$ of the data points corresponding to ionization and $20 \%$ to recombination used in the original scaling law. A separate linear regression analysis shows different dependencies when comparing the scaling laws for ionization, i.e.,

$$
\begin{aligned}
& n_{\mathrm{e}}=10^{7.6 \pm 0.5} \Gamma^{1.6 \pm 0.1} B^{0.82 \pm 0.08} I^{5.2 \pm 0.2}, \\
& T_{\mathrm{e}}=10^{2.5 \pm 0.2} \Gamma^{-0.06 \pm 0.05} B^{0.50 \pm 0.03} I^{-1.0 \pm 0.2}
\end{aligned}
$$

and recombination

$$
\begin{aligned}
& n_{\mathrm{e}}=10^{10.0 \pm 0.7} \Gamma^{-0.5 \pm 0.2} B^{1.0 \pm 0.1} /^{4.8 \pm 0.3}, \\
& T_{\mathrm{e}}=10^{3.9 \pm 0.4} \Gamma^{-1.3 \pm 0.1} B^{0.68 \pm 0.08} /^{-1.3 \pm 0.1} .
\end{aligned}
$$


It can be seen that the plasma parameter dependence on source gas flow depends strongly on the regime of machine operation. On the other hand, the other parameter dependences are more robust. In the ionization phase it is expected that an increase in gas flow will lead to ionization of a large fraction of the added neutrals, causing an increase in plasma density which is reflected by (5a). Conversely, in the recombination phase, the addition of more neutrals principally increases plasma-neutral cooling effects which will decrease the electron temperature and enhance the recombination rate, resulting in a lower plasma density which is reflected by (6a). Hence, Eqs. 4 only summarize global trends in the existing data-set, and the large proportion of measurements with peak $T_{e}>1 \mathrm{eV}$ implies the scaling may be biased towards describing ionization plasmas.

The scaling laws also show that the density in both the ionization phase and recombination phase depends strongly on the current. Analyzing the data in detail shows that this current dependency is not an artifact of the regression analysis but an relationship existing in the actual data. In consecutive discharges in which both the flow $\Gamma$ and magnetic field $B$ are kept constant and only the current $I$ is varied this trend is reproduced independently. It was shown in [15] that $n_{\mathrm{e}}$ at the cascaded arc source exit in Pilot-PSI was linearly proportional to input power at fixed $\Gamma$ and $B$. Assuming similar performance here would indicate that this phenomenon is therefore primarily related to atomic processes and collisions in the plasma beam between the source exit and the downstream TS measurement position. Elucidating this relationship more clearly is a goal of future research.
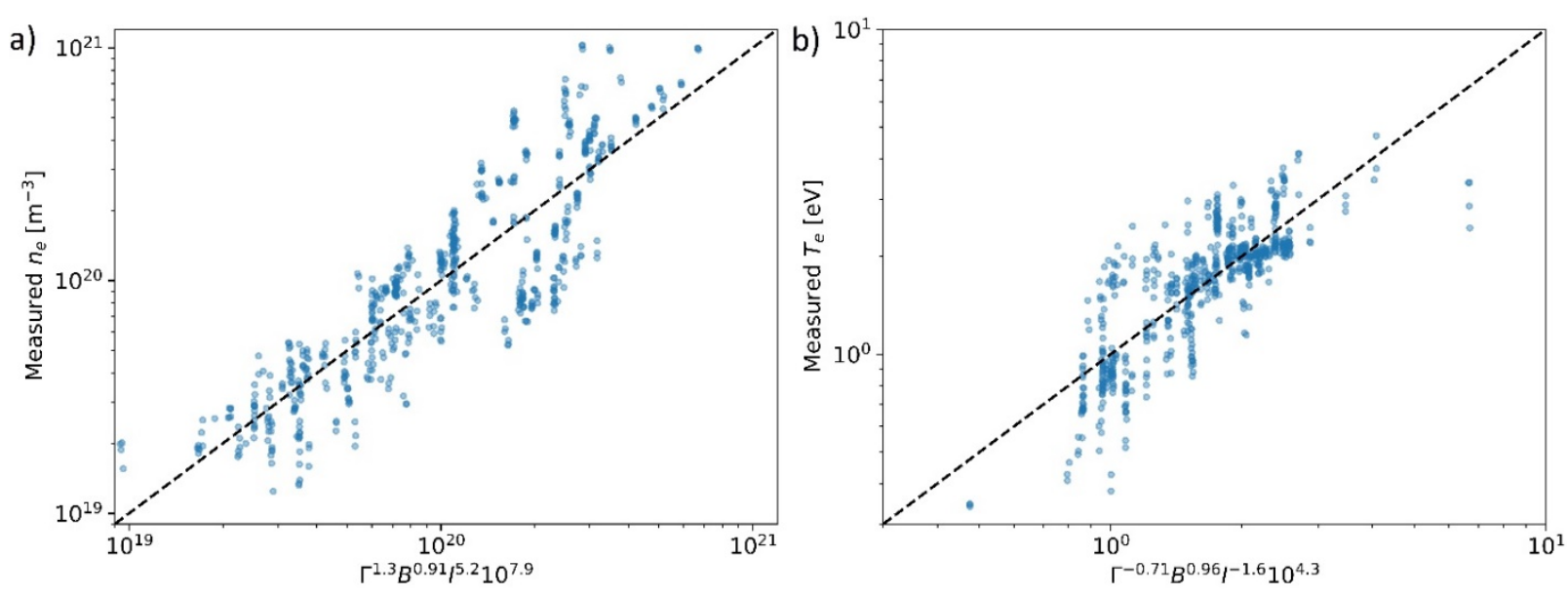

Figure 3. Comparison of scaling law predictions with measured values of electron density (a) and temperature (b). The rms residual between the fit and measurements is $10^{ \pm 0.22}$ for $n_{e}$ and $10^{ \pm 0.12}$ for $T_{e}$.

\subsection{Data fitting using artificial neural networks}

The linear regression in log-log space is in principle fitting to a product of one-term exponential functions in multiple dimension, which cannot map all complexity of the data. In order to be able to do this, alternative methods can be used $[16,17]$. In this study we implemented the artificial neural network (ANN), which is a flexible method that does not require a model a priori, and can map the complexity of data with a high accuracy 
[16]. More information about how the ANN method was applied here can be found in the Appendix. The ANN fitting results in Figure 4 show that by increasing the magnetic field, both $n_{\mathrm{e}}$ and $T_{\mathrm{e}}$ rise, consistent with Eq. $(4 a, b)$. The arc current causes a decrease of $T_{e}$, while it increases $n_{e}$, which is also consistent with Eq. $(4 a, b)$. Hence, the results reveal the same trends as the linear regression (LR) analysis. In contrast, it shows non-linearity in log-log space. The performance of the fitting increased significantly compared to the LR, which is evident from the comparison of the fitting results (LR/ANN) and the measured values, see Figure 5. The relative error (RMSE) dropped from 0.26 to $0.21(24 \%)$ in $T_{\mathrm{e}}$ and from 0.48 to $0.43(12 \%)$ in $n_{\mathrm{e}}$. This obtained RMSE between the data and the ANN fit is still larger than the observed error in reproducibility of the data by a factor $\sim 5$, which means that either the present form of ANN is not much more effective in describing the plasma response to machine settings in all regimes of machine operation compared to a standard scaling law, or the scatter in the data is strongly underestimated.
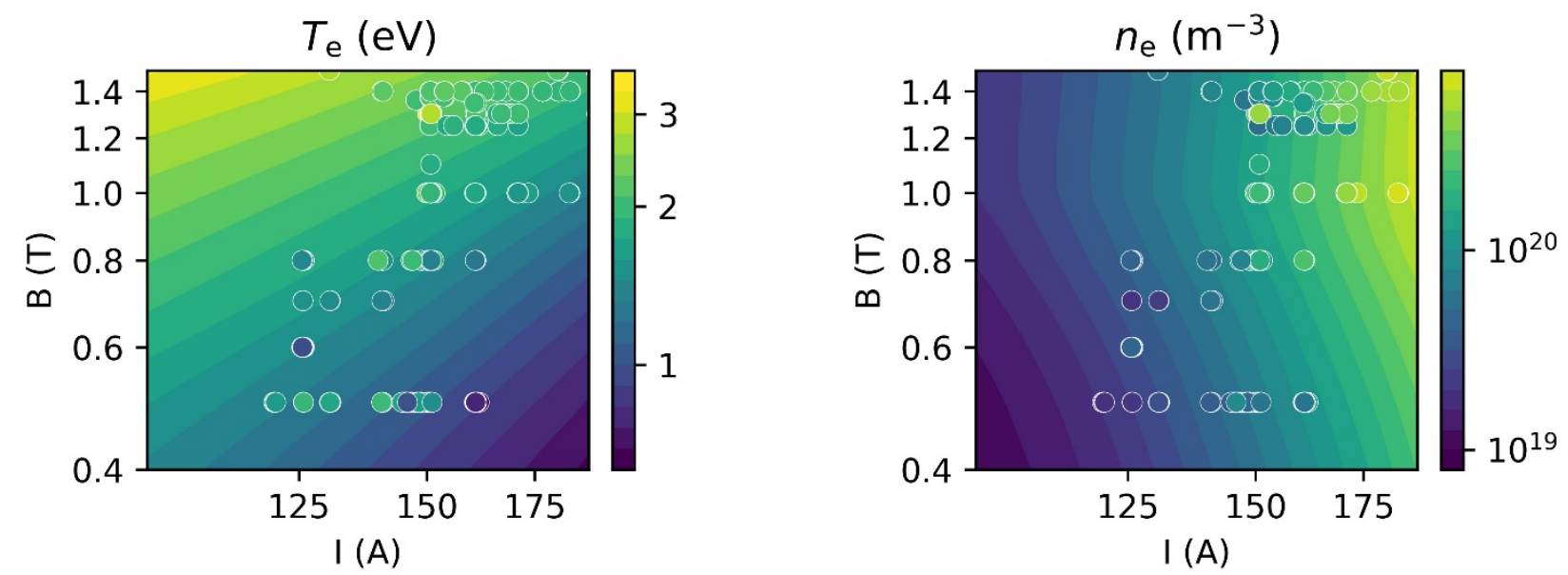

Figure 4. The electron temperature $\left(T_{\mathrm{e}}\right)$ and density $\left(n_{\mathrm{e}}\right)$ as function of the magnetic field $B$ and arc current $I$ at constant gas flow (5 s/m). The measured values are plotted along the fitted data (filled contour levels).
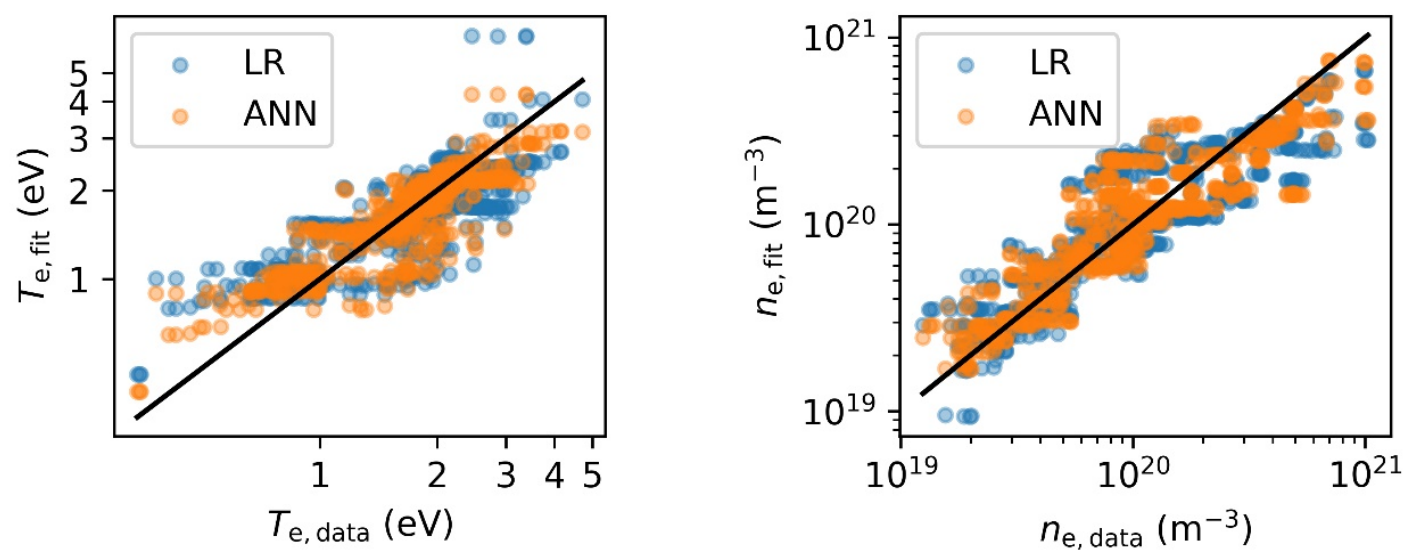

Figure 5. The fitted electron temperature and density $\left(T_{\mathrm{e}, \mathrm{fit}} / n_{\mathrm{e}, \text { fit }}\right)$ as function of the measured values $\left(T_{\mathrm{e}, \mathrm{data}} / n_{\mathrm{e}, \mathrm{data}}\right)$. 


\subsection{Characterisation of ELM-like pulses in Magnum-PSI}

Magnum-PSI is equipped with a high-repetition rate ELM replication system [2]. On top of the steady state source current, a capacitor bank system, consisting of 28 identical sections, can be discharged through the plasma source to produce transient plasma pulses with high heat and particle fluxes. This capacitor bank system allows for high repetition pulses $(>100 \mathrm{~Hz})$ and flexible pulse shaping by varying the timing and voltage of the individual capacitor sections. To characterize these pulses in Magnum-PSI, a systematic scan in the machine parameters was performed. The magnetic field $B$ was varied between 0.8 and $1.2 \mathrm{~T}[0.8,1.0$ and $1.2 \mathrm{~T}]$, the source gas flow $\Gamma$ between 8 and $14 \operatorname{sim}[8,10,12$ and $14 \mathrm{slm}]$ and the capacitor bank voltage $U$ between 400 and $2000 \mathrm{~V}$ (in steps of $200 \mathrm{~V}$ ), providing a database of 46 different pulses types. Only one capacitor section was used per pulse. Using multiple sections per pulse would enable pulse shaping and even more intense pulses. The temperature and density of each pulse type was measured using time resolved TS [9], measuring a TS spectrum at 50 different timings (relative to the pulse trigger) on a train of 50 identical pulses. The target was retracted far back from the TS position to avoid influencing the pulse characteristics.

The shape of the pulses was found to be independent of $B$ and $\Gamma$, but to vary significantly with the capacitor bank voltage $U$. Figure 6 shows the pulse shapes for both density and temperature for three different voltages $U=400,1200$ and $2000 \mathrm{~V}$. The density pulses are more elongated in time than the temperature pulses, with higher voltages giving longer pulses. The maximum achieved temperature in each pulse does not necessarily coincide with the maximum density.

The maximum temperatures and densities achieved in the database varied from 3-18 eV and 1.5-2.8 $10^{21} \mathrm{~m}^{-3}$ respectively. Unlike the steady state measurements, the ELM-like pulses do not exhibit a clear power dependence with respect to the capacitor bank voltage $U$. Hence, a scaling law is unsuitable to reflect the machine parameter space. 

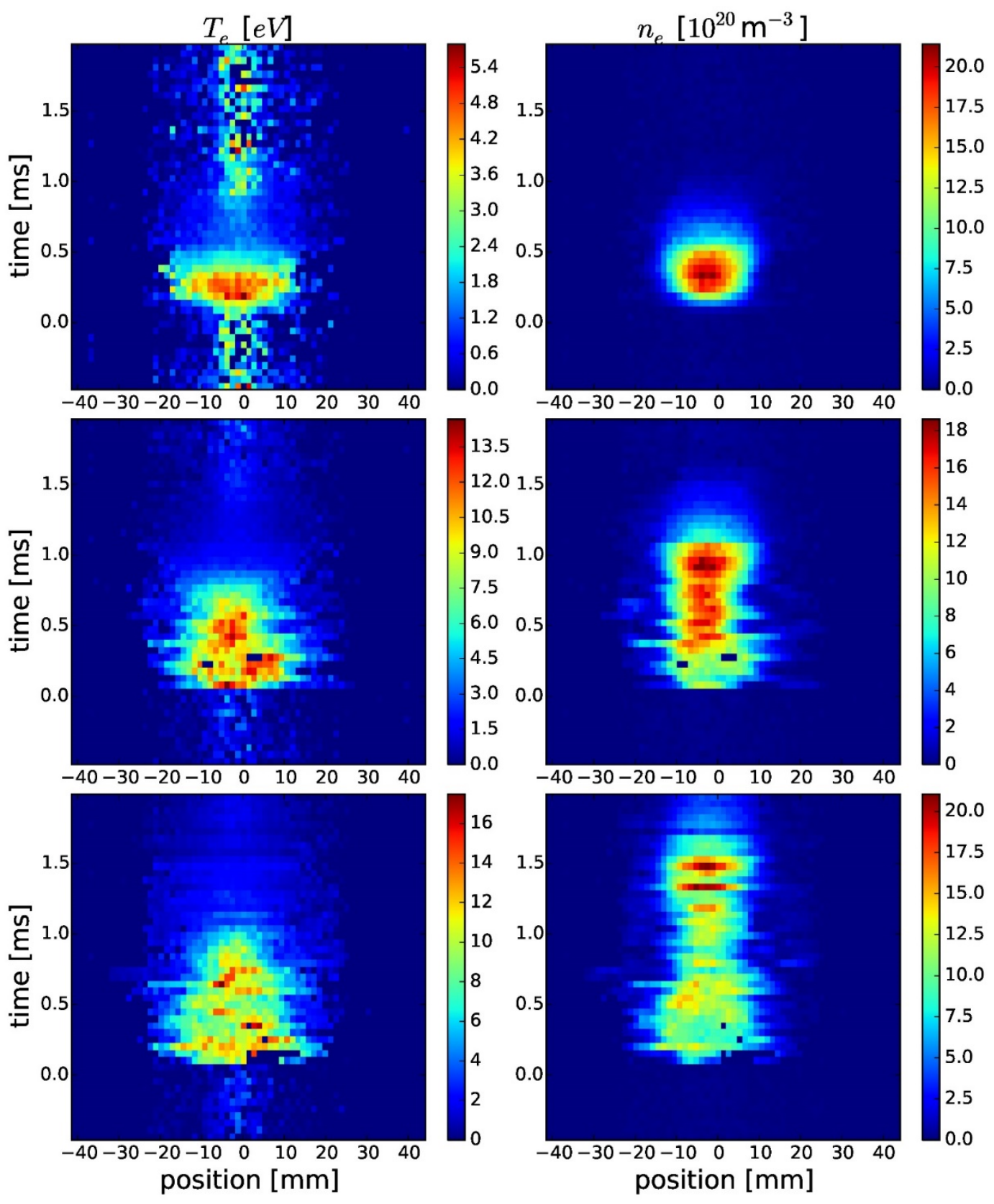

Figure 6. Time resolved temperature (left) and density (right) of ELM-like pulses in Magnum-PSI measured using TS. Three pulses with the same $B$ and $\Gamma(1 \mathrm{~T}, 10 \mathrm{slm}$ ) but different capacitor voltage (top row: $400 \mathrm{~V}$, middle row: $1200 \mathrm{~V}$, bottom row: $2000 \mathrm{~V}$ ) show a large variation in pulse shape and duration.

\section{High fluence experiments}

The updated capabilities of Magnum-PSI enable new venues of exploration, at higher fluence, higher fluxes and with new diagnostic capabilities than were previously possible. As an example of the high-fluence capabilities a water-cooled monoblock chain identical to that used in [18] was exposed to a variety of plasma conditions in Magnum-PSI, including $\mathrm{H}, \mathrm{He}, \mathrm{D}$ and $\mathrm{D}+\mathrm{He}$ plasma (89:11 gas ratio). In the longest (D) exposure this target 
received a fluence of $1.01 \times 10^{30} \mathrm{~m}^{-2}$ while also matching the expected DT-phase operating temperature of $1200^{\circ} \mathrm{C}$ [19]. This enabled for the first time experiments to probe lifetime performance of the monoblock, comparable to ITER conditions and with similar electron and ion temperatures as would be expected at the strike points during operation. The total fluence was equivalent to around 12 months of ITER operation [19], achieved in 19.7 hours (Figure 7). The full results will be published elsewhere.

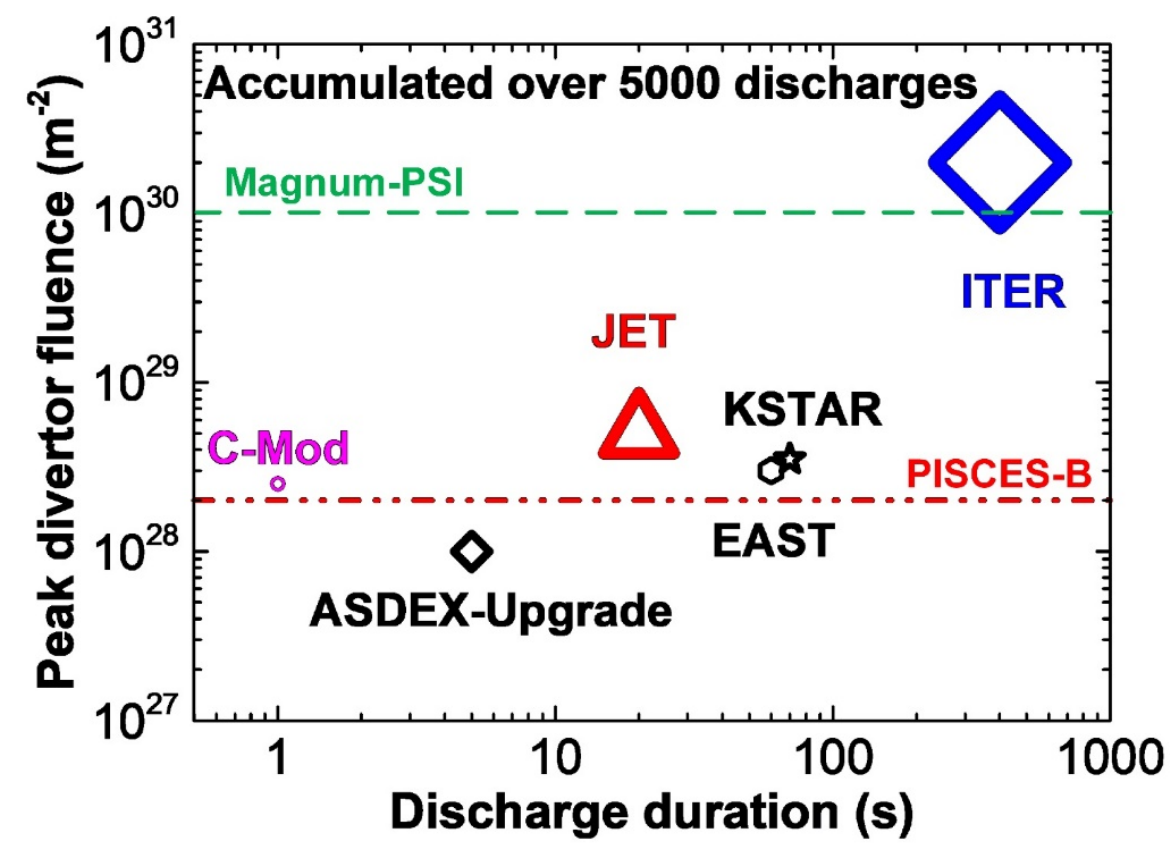

Figure 7. Peak ion fluence in the divertor for several different tokamaks over 5000 typical plasma discharges. The red dotdashed line represents the highest reported fluence in literature achieved in any linear device [20] while the green dashed line represents the fluence in Magnum-PSI reported here. Adapted with permission from [21] C 2018 ITER Organization.

\section{Conclusions}

The operational regime of the linear plasma generator Magnum-PSI has been explored after the arrival of the superconducting magnet. With the new superconducting magnet, electron densities up to $10^{21} \mathrm{~m}^{-3}$ and electron temperatures up to $4.7 \mathrm{eV}$ can be reached leading to particle fluxes up to $10^{25} \mathrm{~m}^{-2} \mathrm{~s}^{-1}$ and heat fluxes up to 50 $\mathrm{MWm}^{-2}$. This, combined with the ability to maintain these conditions for hours on end, make the facility highly relevant for plasma wall interaction studies for fusion reactors such as ITER and DEMO. Linear regression and artificial neural network analysis have been used to gain insight in the general behavior of plasma conditions as a function of machine settings. Both techniques yielded similar results. On top of the steady state operation, a capacitor bank system can be discharged through the plasma source to produce transient plasma pulses with high heat and particle fluxes. 


\section{Appendix}

The ANN was set up using the TensorFlow toolbox in Python [22]. Essentially, ANNs consists of connected nodes, so-called neurons, arranged in several layers. Each neuron is activated by a non-linear function $\varphi$ (ReLu in this paper [23]) working on the sum of weighted inputs $\boldsymbol{w} \cdot \boldsymbol{x}$ from all neurons of the preceding layer plus a bias $b$ (i.e., $o=\varphi(\boldsymbol{w} \cdot \boldsymbol{x}+b))$. Its output $o$ is transmitted to all neurons from the succeeding layer. In this manner, input parameters in the first layer, are transmitted through the ANN to generate predicted parameters $P$ at the output layer. In this study, an ANN with two hidden layers of 5 neurons (56 free parameters of weights and biases) was trained by supplying the network with measurement data of Magnum-PSI (inputs $I_{\mathrm{m}}$ and outputs $O_{m}$ ). The training data was used to adjust the weights and biases in each neuron using the Adam optimizer [24] (a gradient descent method including learning rate scaling and momentum addition), which minimize the loss function $E=\frac{1}{2}\left|O_{\mathrm{m}}-P\left(I_{\mathrm{m}}\right)\right|^{2}$ with a learning rate of 0.0005 . In order to avoid overfitting, L2 regularization [25] was applied with a regularization constant of 0.001 . Moreover, to assess the quality of the fitting, the dataset of Magnum-PSI was split in two parts, a training set and validation set of $70 \%$ and $30 \%$ of the total data, respectively, i.e. a deviating root mean squared error (RMSE) for the validation set compared to the training set indicates overfitting. The training was performed on 50.000 batches of 50 random samples each. Lastly, the concept of ensemble averaging of networks [26] was applied to increase the fitting performance, and to obtain an estimate of confidence across the parameter domain. The average was defined as $\bar{y}=\sum_{i=0}^{n} w_{i} x_{i} / \sum_{i=0}^{n} w_{i}$, in which $w_{i}=1 / \sigma_{i}^{2}$, and $\sigma_{i}$ is the RMSE of each network, and the variance is $\sigma_{\bar{y}}=1 /(n-$ 1) $\cdot \sum_{i=0}^{n} w_{i}\left(x_{i}-\bar{x}\right)^{2} / \sum_{i=0}^{n} w_{i}$. For each output parameter, 25 ANNs were trained, and before the training of a new network, the data was shuffled and regrouped in a new training and validation set.

\section{Acknowledgements}

We acknowledge Jonathan van den Berg and Karel van de Plassche for their input on ANNs. The actively-cooled monoblock chain was supplied by the ITER Organization and F4E as part of a Eurofusion-funded experiment to investigate the effect of ITER-relevant fluences on the properties of tungsten. This work has been carried out within the framework of the EUROfusion Consortium and has received funding from the Euratom research and training programme 2014-2018 and 2019-2020 under grant agreement No 633053. The views and opinions expressed herein do not necessarily reflect those of the European Commission. DIFFER is a partner in the Trilateral Euregio Cluster TEC.

\section{References}

[1] G.M.W. Kroesen, D.C. Schram, M.J.F. van de Sande, Plasma Chem. and Plasma Proc. 10 (1990) 49, https://doi.org/10.1007/BF01460447.

[2] T W Morgan et al, Plasma Phys. Control. Fusion 56 (2014) 095004, https://doi.org/10.1088/0741-3335/56/9/095004.

[3] Ch. Linsmeier et al, Nucl. Fusion 57 (2017) 092012, https://doi.org/10.1088/1741-4326/aa4feb. 
[4] S. Brezinsek et al, Nucl. Fusion 57 (2017) 116041, https://doi.org/10.1088/1741-4326/aa796e.

[5] H.J.N. van Eck, W.R. Koppers, G.J. van Rooij, W.J. Goedheer, R. Engeln, D.C. Schram, N.J. Lopes Cardozo and A.W. Kleyn, J. Appl. Phys. 105 (2009) 063307, https://doi.org/10.1063/1.3086622.

[6] R. Perillo et al, Nucl. Mater. Energy 19 (2019) 87, https://doi.org/10.1016/j.nme.2019.02.018.

[7] H.J.N. van Eck, A.W. Kleyn, A. Lof, H.J. van der Meiden, G.J. van Rooij, J. Scholten and P.A. Zeijlmans van Emmichoven, Appl. Phys. Lett. 101 (2012) 224107, https://doi.org/10.1063/1.4768302.

[8] H.J.N. van Eck, H.H.J. ten Kate, A.V. Dudarev, T. Mulder, and A. Hervé, IEEE Trans. Appl. Supercond. 28 (2018) 1, https://doi.org/10.1109/TASC.2017.2779510.

[9] H.J. van der Meiden et al, Rev. Sci. Instrum. 83 (2012) 123505, https://doi.org/10.1063/1.4768527.

[10] H.J. van der Meiden, Plasma Phys. Control. Fusion 52 (2010) 045009, https://doi.org/10.1088/07413335/52/4/045009.

[11] G.G van Eden, M.L Reinke, S. Brons, G. van der Bijl, B.J.M Krijger, R. Lavrijsen, S.P Huber, R. Perillo, M.C.M van de Sanden, T.W Morgan, Nucl. Fusion 58 (2018) 106006, https://doi.org/10.1088/1741-4326/aad0a9.

[12] Piip et al, J. Nuclear Mater. 463 (2015) 919-922, https://doi.org/10.1016/j.jnucmat.2014.11.017.

[13] P.C. Stangeby, The Plasma Boundary of Magnetic Fusion Devices, Taylor \& Francis Group, 2000.

[14] T.W. Morgan, M.A. van den Berg, G. De Temmerman, S. Bardin, D.U.B. Aussems, R.A. Pitts, Nucl. Fusion 57 (2017) 126025, https://doi.org/10.1088/1741-4326/aa8109.

[15] W.A.J. Vijvers, C.A.J. van Gils, W.J. Goedheer, H.J. van der Meiden, D.C. Schram, V.P. Veremiyenko, J. Westerhout, N.J. Lopes Cardozo and G. J. van Rooij, Phys. Plasmas 15 (2008) 093507, http://dx.doi.org/10.1063/1.2979703.

[16] B.J. Braams, W. Jilge, K. Lackner, Nucl. Fusion. 26 (1986) 699, https://doi.org/10.1088/0029-5515/26/6/001.

[17] J. van den Berg, I. Abramovic, N. Lopes Cardozo, D. Moseev, Rev. Sci. Instrum. 89 (2018) 083507, https://doi.org/10.1063/1.5035416.

[18] Th. Loewenhoff, S. Bardin, H. Greuner, J. Linke, H. Maier, T. W. Morgan, G. Pintsuk, R.A. Pitts, B. Riccardi and G. De Temmerman, Nucl. Fusion 55 (2015) 123004, https://doi.org/10.1088/0029-5515/55/12/123004.

[19] R.A. Pitts et al, J. Nucl. Mater. 438 (2013) S48-S56, https://doi.org/10.1016/j.jnucmat.2013.01.008.

[20] R.P. Doerner, M.J. Baldwin, T.C. Lynch and J.H. Yu, Nucl. Mater. Energy 9 (2016) 89, https://doi.org/10.1016/j.nme.2016.04.008.

[21] G. De Temmerman, T. Hirai and R.A. Pitts, Plasma Phys. Control. Fusion 60 (2018) 044018, https://doi.org/10.1088/1361-6587/aaaf62.

[22] M. Abadi et al, TensorFlow: A System for Large-Scale Machine Learning TensorFlow: A system for large-scale machine learning, 12th USENIX Symp. Oper. Syst. Des. Implement. (2016) 265-284.

[23] V. Nair, G.E. Hinton, Rectified Linear Units Improve Restricted Boltzmann Machines, Proc. 27th Int. Conf. Mach. Learn. (2010) 807-814.

[24] D.P. Kingma, J. Ba, Adam: A Method for Stochastic Optimization, Proceedings of the $3^{\text {rd }}$ International Conference on Learning Representations (ICLR) (2014) 1-15.

[25] Y. Bengio, Practical recommendations for gradient-based training of deep architectures, Neural networks: Tricks of the trade - Springer (2012) 437-478.

[26] S. Hashem, Neural Netw. 10 (1997) 599, https://doi.org/10.1016/S0893-6080(96)00098-6. 